\title{
An Optimized Hybrid Filter designing for Speckle and Gaussian Noise Reduction in Ultra-Sound Images
}

\author{
Archana Madan, Daljit Singh
}

\begin{abstract}
Image which is a visual perception of a scene or something is a set of pixels that have certain values which appear in the form of colors to view that particular set of pixel as image. Image contains information about whatever is being depicted in the picture and hence it can be said as a useful source for storing or conveying information. Image noise is apparent in image region with low signal level, such as shadow region or under exposed images. The work presents the concept of noising and denoising in a digital image. Noise is a kind of disturbance that occurs in the channel at the time of transmission. Image denoising is the procedure of improving true images from the noisy images. For ages researchers have been proposing several techniques that were used to remove the noise from the image. Image denoising is the procedure of improving true image from the noisy image. At the time of such process it is difficult to reduce noise. Owing to this difficulty, numerous denoising models have been proposed [4]. The paper presents the reduction of speckle and Gaussian noise in the biomedical ultrasound images. In the proposed work, the Butterworth filter is applied for filtering the noisy image and then the coefficients are optimized by using the firefly algorithm mechanism to remove noise that occurs at the time of transmission and then it is hybrid with the Weiner filter. Experiments have been performed to check the performance of the proposed technique. The results are analyzed quantatively using PSNR and SSIM. The results are also evaluated on other performance parameters such as BER, MSE and fitness on speckle as well as on Gaussian noise.
\end{abstract}

Index Terms: Speckle Noise, Gaussian Noise, Denoising, Butterworth filter, Firefly Algorithm, Digital Image, Ultrasound Image

\section{INTRODUCTION}

To create the image of the human body the process called as medical imaging is done. In medical imaging, various techniques are used that help to create the image. These images are useful for various clinical applications such as in radiography the diagnosis is performed using these images [1]. The surgeons especially the radiographers need the image of high quality for diagnosis purpose. There are different types of medical imaging :
a. Computed Tomography (CT)
b. Magnetic Resonance Imaging (MRI)
c. Ultrasound

In this era most of the data travels through internet due to which the chances of noise in the data increases. The carrier signals are more sensitive to these noisy signals.

Revised Manuscript Received on June 15, 2019.

Er. ArchanaMadan, Former Senior Engineer, Max Speciality Films Limited, India.

Er. Daljit Singh, Assistant Professor - Guru Nanak Dev Engineering College, Department of Electronics and Communication Engineering, India.
The noise in the signals take place due to the introduction of the unwanted information in the signals that destruct the original information sometimes. Noise is the kind of disturbance or irrelevant data or sound in the actual data which leads to the loss of data to be transferred at the destination [2]. The disturbance to the signal can happen due to external network links like hum that suddenly enters to your transmission channel and perform data tempering in various ways. None of the data is pure i.e. each and every data comprises of some noise always. But if it crosses the threshold limit then it is considered as noise or disturbance [3]. The images can be affected by the noise in following manner :

1. Unwanted spots or objects can be seen in the image.

2. The suffered image will face the loss of data.

3. The quality of the received image will be degraded in comparison to original image.

4. The original image can be lost fully if the level of the noise is increased by the value of the threshold.

5. The noisy image cannot be reused anywhere

6. The noisy image will suffer from lack of visibility or quality

Image can suffer from assorted types of noise listed below :

1. Gaussian Noise

2. Salt and Pepper Noise

3. Shot Noise

4. Quantization Noise

5. Filmgrain Noise

6. Anisotropic Noise

7. Periodic Noise

8. Speckle Noise

In this paper, the scope is restricted to Speckle and Gaussian Noise as these are most likely noises that occurs in medical images.

Speckle Noise : It is the characteristic effect seen in the ultrasound images that contribute to the visual noise. The image of a relatively uniform object with many scattering sources within a resolution cell will have pixels that vary randomly with position due to constructive and destructive interference.

$F(g)=\frac{g^{\alpha-1}}{(\alpha-1) ! a^{\alpha}} e^{\frac{-g}{a}}$

- It is a multiplicative noise which is in direct proportion to the local grey level in any area. 
- The signal and noise are statistically independent.

- The sample mean and variance of a single pixel are equal to the mean and variance of local area.

Gaussian Noise : It exists with the probability density function i.e. PDF and also refereed as Gaussian distribution. In this Gaussian distribution refers to the value that noise can take over. In order to calculate PDF of Gaussian filter following formulae has been used.

$\mathrm{p}_{\mathrm{G}}(\mathrm{z})=\frac{1}{\sigma \sqrt{2 \pi}} \mathrm{e}^{-\frac{(\mathrm{x}-\mu)^{2}}{2 \sigma^{2}}}$

\section{TECHNIQUES USED FOR NOISE REDUCTION}

Rajesh Mohan R, (2016) developed a novel approach to get rid of speckle noise in microwave images. In the paper by Rajesh, algorithm by Liu[14], Technique represented the speckle noise reduction algorithm which worked by combining the adaptive wavelet soft thresholding operation along with Partial Differential Equation (PDE). The purpose behind using the PDE was to remove the noise that remains left after applying the adaptive wavelet thresholding method. He proposed an approach (referring as traditional approach) represents a noise removal by combining the spatial and frequency domain methods. At initial stage the wiener filters was applied in spatial domain as a pre-processing mechanism and soft thresholding wavelet transform was implemented in frequency domain. Then logarithmic transformation was applied by considering the multiplicative model to filtered image. The standard deviation equation was utilized corresponding to each decomposition level [2].

\section{PROBLEM FORMULATION}

- At the time of acquisition, noise may enter into an image which distorts the actual quality of the signal. Considering this fact, in the existing work, Weiner filter has been used to remove the noise from the image and adaptive wavelet thresholding has been used for filtration [7].

- From the applications of this technique, it has concluded that the value of the coefficient remained fixed until the execution. In other words, the best value has acquired and the coefficients remained constant with this value.

- Accordingly, this method limits the process of filtration and can remove the noise at some extent which means that it does not consider the amount of noise having in the image [8]. Consequently, noise cannot be removed completely from the image.

- Secondly, the applied wavelet thresholding technique is not capable in reserving the edge of the images due to which the problem of lacking shift invariance occurs. In order to exclude these issues, there is a requirement of replacement of this technique with the advanced methods..

\section{PRESENT PROPOSED WORK}

1. The speckle noise and Gaussian noise are two main type of noises that has been considered in present work for implementation

2. The present work comprised of a novel approach that has introduced in this work where wiener filter has been replaced with the Butterworth filter. Because the Butterworth filter design the coefficients and does not remain them constant. The values of the coefficients varies with each iteration. In Weiner filter there is no adaptive property whereas in butterworth filter it can be changed according to the data input.

3. The acquired coefficient's value will be optimized using the firefly algorithm that rule out the lack of shift invariance in the existing work. The firefly algorithm worked continuously over the values until it does not attained the best optimum solution for the problem and keep on varying the coefficient's value till the deduction of noise from the image.

4. As a result, it can say that proposed algorithm have the capability to retain the process till the removal of noise from the image entirely.

The proposed image denoising is developed by using filtration and optimization techniques. These specific techniques are as follows:

\section{Butterworth Filter}

Butterworth filter helps in maintaining the flat and static output response. The butterworth LPF operates somewhat in similar manner to the ideal filter without few steps discontinuous model [6]. In this filter the transfer function with $\mathrm{n}$ order and predefined cut off frequency locus at a distance from the origin can be described with the help of following mathematical equation

$$
\begin{aligned}
& H(a, b)=\frac{1}{1+\left[\frac{I(a, b)}{I_{0}}\right]^{2 n}} \ldots \ldots \ldots \ldots \ldots \ldots \text { (iii) } \\
& I(a, b)=\left(a^{2}+b^{2}\right)^{1 / 2} \ldots \ldots \ldots \ldots \text { (iv) }
\end{aligned}
$$

\section{Firefly Optimization}

Firefly algorithm is one of the optimization algorithms developed by Yang. Main characteristics of the firefly algorithms are as follows:

1. Despite their sex, they get attracted to other flies. Thus fireflies are unisex.

2. Fireflies that have less brightness as compared to other flies in such case these are attracted to brighter ones. Attractiveness and brightness of the firefly depend upon the distance they maintain as it will be decreased with the increase in distance. Moreover, fireflies do not find brighter fireflies in their path when they start moving randomly. 
3. Lastly brightness of a firefly is fully dependent on the setting of the objective functions.

Consequently, firefly algorithm is based on these two concepts as a variation of the light intensity and formulation of attractiveness whereas attractiveness of a firefly can be formed by the brightness of a firefly. And it has been treated as an objective function of the optimization problem. As firefly's attractiveness is directly propositional to the light intensity which can be seen by adjacent fireflies.

The variation of attractiveness with the distance can be evaluated by using

$$
\beta(r)=\beta_{0} e^{-y r^{2}}
$$

The movement of firefly I with less brightness is towards the another firefly $\mathrm{j}$ is determined by
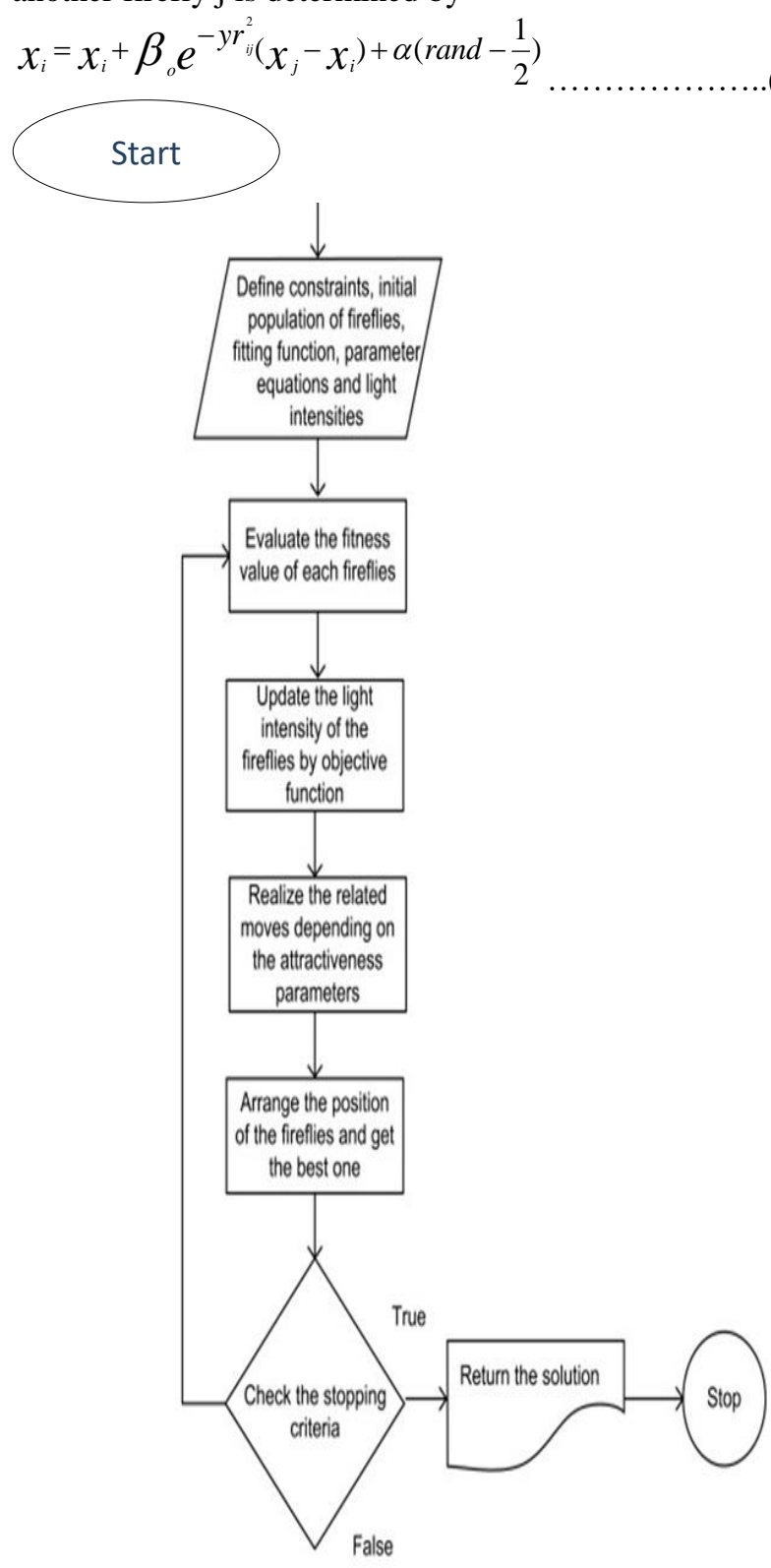

Fig 1 : Flow Diagram of firefly Optimization Technique

Methodology : The methodology for proposed work is as below :
1. Browse the image

2. Add Noise

3. Apply Filters

4. Optimization of coefficients

5. Performance Evaluation

6. Comparison Study

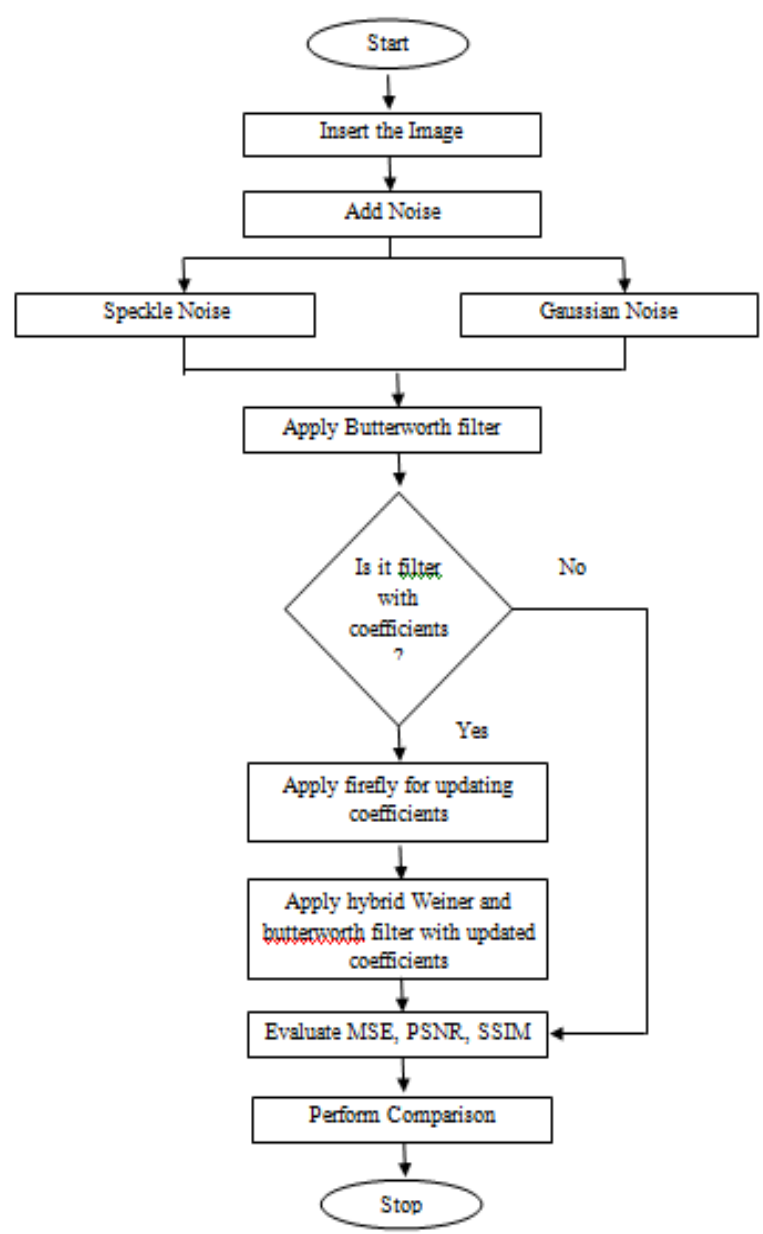

Fig 2 : Flow Diagram of proposed work

\section{RESULTS}

In this work total five images are used for implementation i.e. Lena, Cameraman, Monarch, House and Ultrasound Image. This section defines the results in the form of graphs that are obtained after implementing the proposed work. The parameters are selected on the basis that filter coefficients should be those which provide more PSNR and less MSE. The performance is measured in terms of PSNR, SSIM, fitness, MSE and BER shown in Table 1 and Table 2. The plots for these parameters in case of speckle as well as Gaussian noise are shown in Fig 5, Fig 6, Fig 7, Fig 8 and Fig 9.Then for the purpose of proving the proficiency of proposed work, the contrast is portrayed among the present study, algorithm given by Liu [14] and traditional work [2] shown in Table 3. 


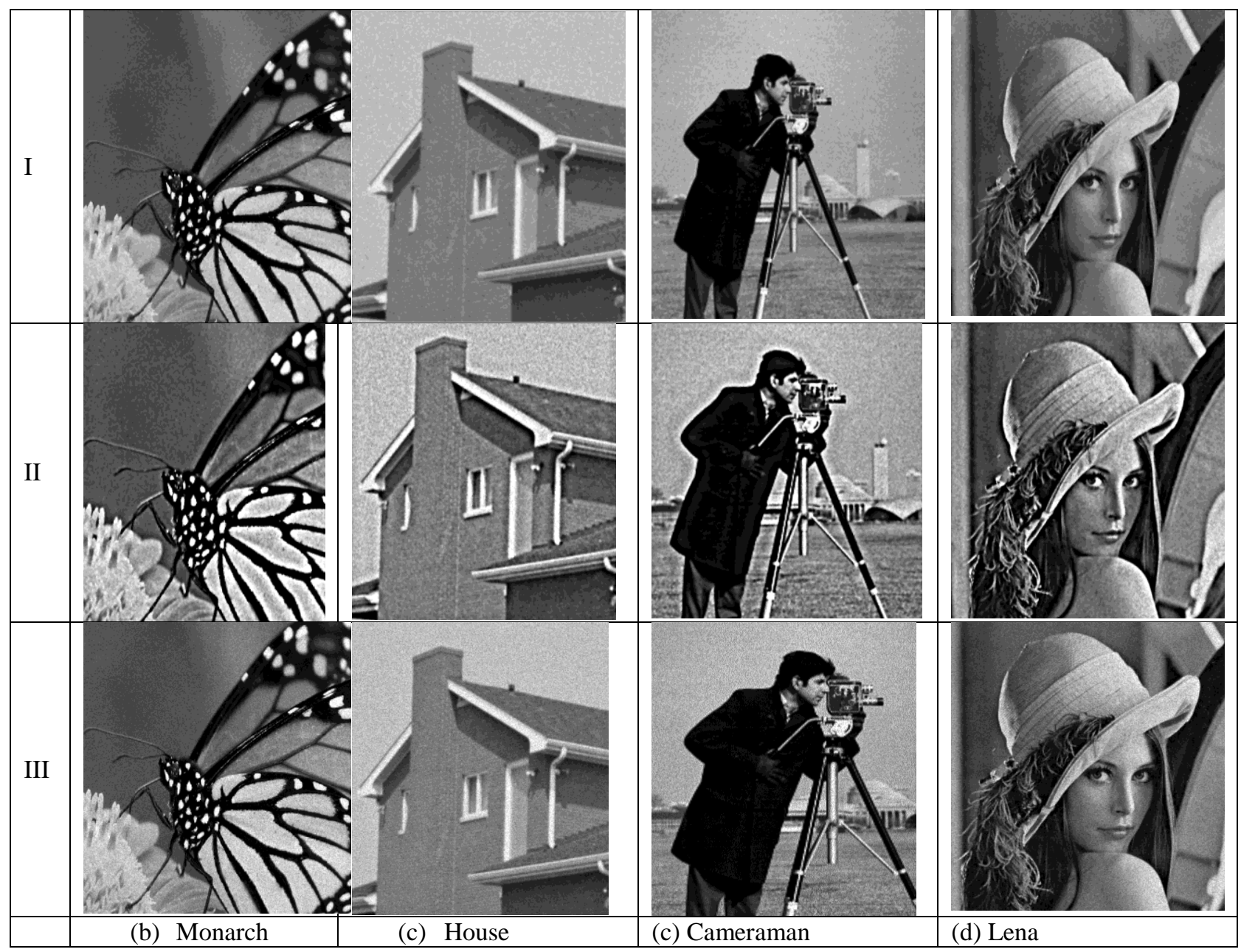

Fig 3 :

I) The figure represents the original images of (a) Monarch (b) House (c) Cameraman (d) Lena.

II) The figure depicts the noisy images that are created by adding the speckle noise in the original images. After adding noise, some small spots can be located on the images.

III) The figure portrays the filtered images of Monarch, House, Cameraman and Lena. The filtered images are generated by using the Butterworth filters and it is observed that the resultant images are smoother than the noisy images.

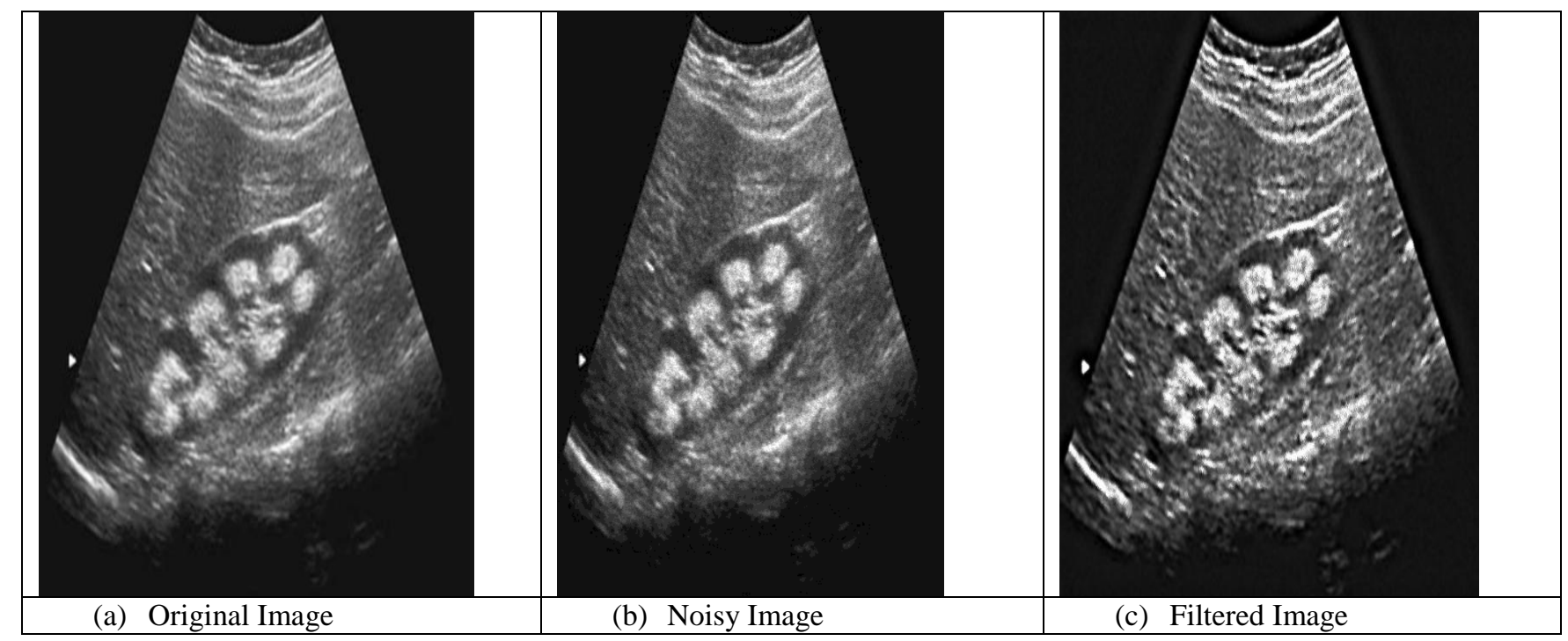

Fig 4: Ultrasound images

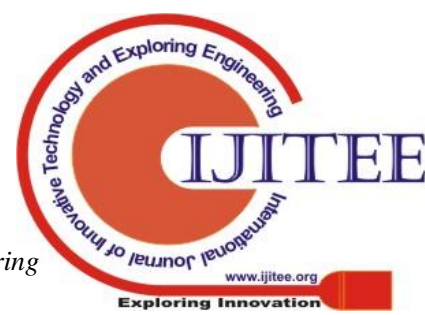


International Journal of Innovative Technology and Exploring Engineering (IJITEE) ISSN: 2278-3075, Volume-8, Issue-9S, July 2019

\begin{tabular}{|c|c|c|c|c|c|c|}
\hline \multirow{2}{*}{ S. NO } & \multirow{2}{*}{ Techniques } & Lena & Cameraman & Monarch & House & UltraSound Image \\
\hline & & \multicolumn{5}{|c|}{ Speckle Noise } \\
\hline 1. & PSNR & 32.7286 & 33.3548 & 34.4113 & 32.1420 & 34.7177 \\
\hline 2. & Fitness & 5.6172 & 7.7419 & 5.5504 & 9.4955 & 6.5 \\
\hline 3. & SSIM & 0.88171 & 0.88051 & 0.91523 & 0.9022 & 0.96 \\
\hline 4. & BER & 0.03055 & 0.0299 & 0.0311 & 0.02906 & 0.27 \\
\hline 5. & MSE & 34.6913 & 30.0331 & 39.7082 & 23.5478 & 13.5 \\
\hline
\end{tabular}

Table 1 : Performance Parameters PSNR, Fitness, SSIM, BER and MSE for the general images for Speckle Noise

\begin{tabular}{|c|c|c|c|c|c|c|}
\hline \multirow{2}{*}{ S. NO } & \multirow{2}{*}{ Techniques } & Lena & Cameraman & Monarch & House & UltraSound Image \\
\hline & & \multicolumn{5}{|c|}{ Gaussian Noise } \\
\hline 1. & PSNR & 32.7253 & 33.3616 & 34.4106 & 32.0962 & 23.5516 \\
\hline 2. & Fitness & 5.5975 & 7.7631 & 5.5053 & 9.5076 & 9.5 \\
\hline 3. & SSIM & 0.86406 & 0.87229 & 0.88297 & 0.88009 & 0.82 \\
\hline 4. & BER & 0.0305 & 0.0299 & 0.0311 & 0.0290 & 0.32 \\
\hline 5. & MSE & 34.7177 & 29.9861 & 40.1292 & 23.5516 & 57.7 \\
\hline
\end{tabular}

Table 2 : Performance Parameters PSNR, Fitness, SSIM, BER and MSE for the general images for Gaussian Noise
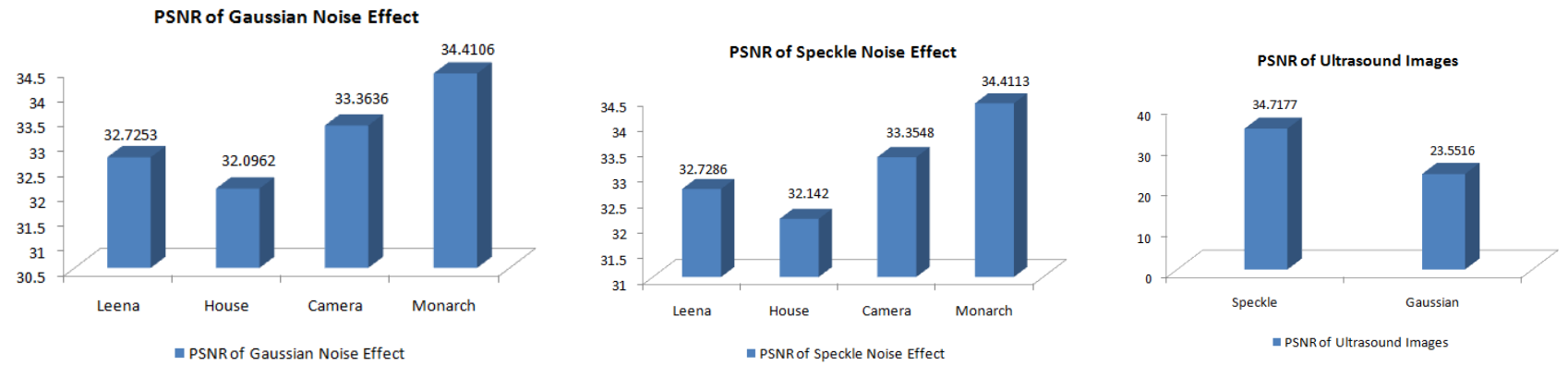

Fig 5 : Plots for PSNR of the present work for Speckle and Gaussian Noise
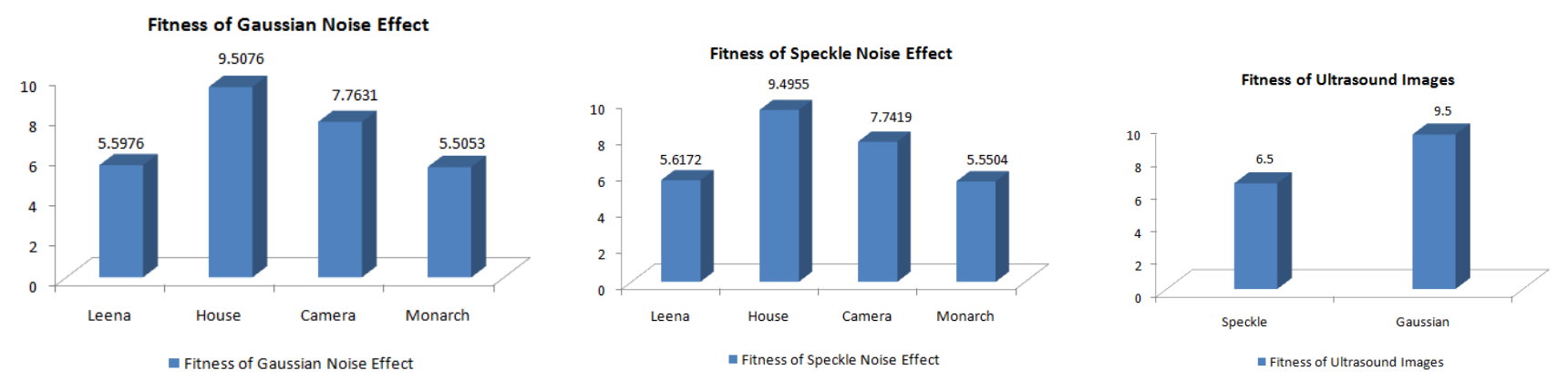

Fig 6 : Fitness of present work for Speckle and Gaussian Noise 

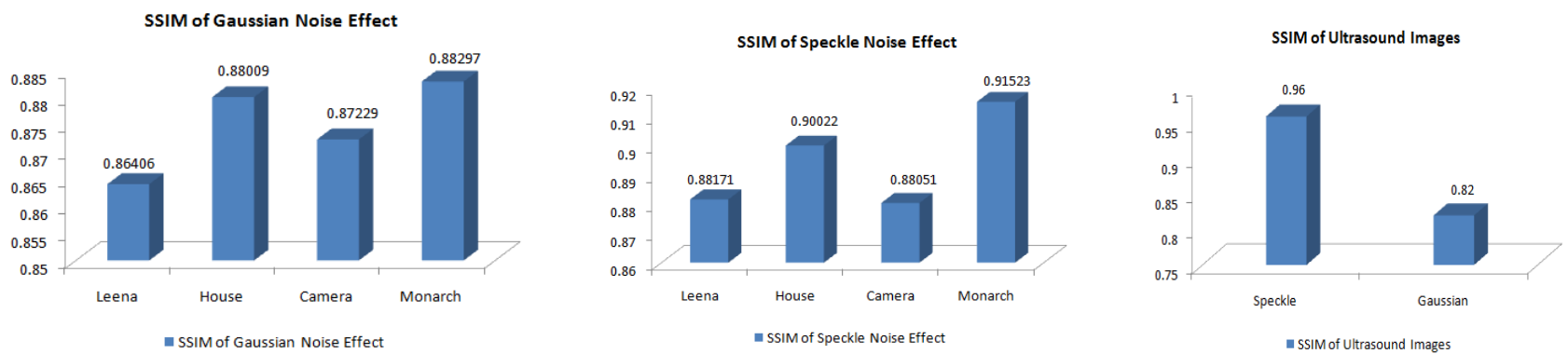

Fig 7 : Plots for SSIM for proposed work for Speckle and Gaussian Noise
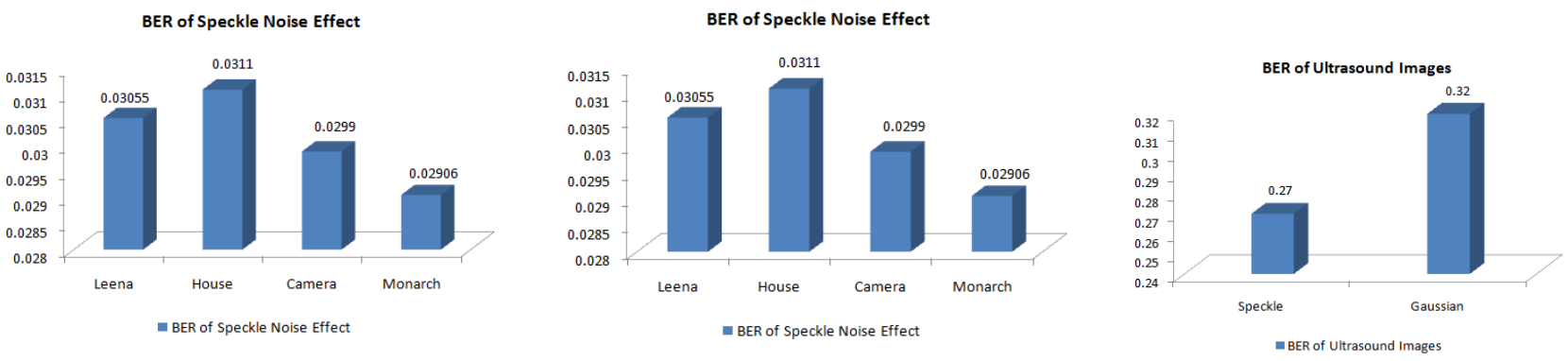

Fig 8 : Plots for BER of proposed work for Speckle and Gaussian Noise
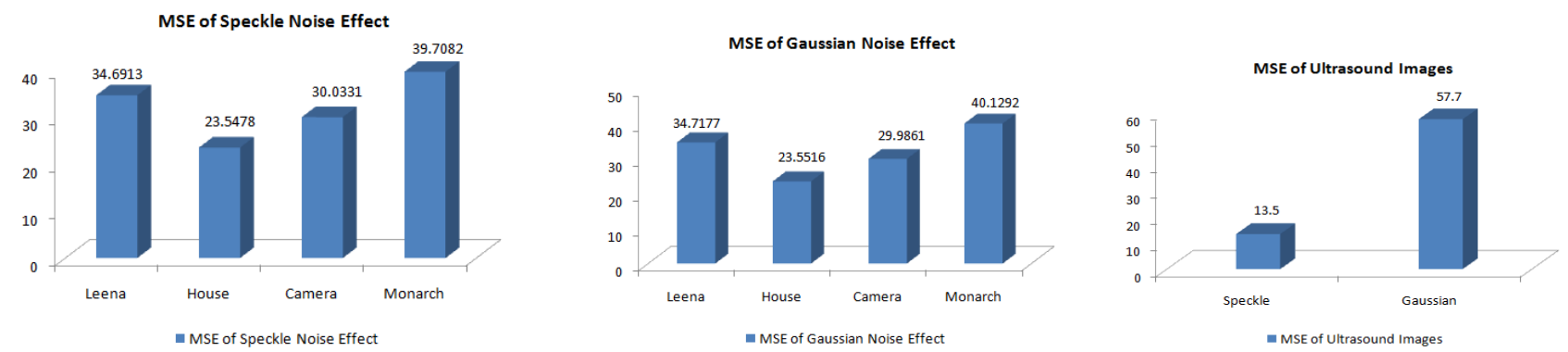

Fig 9 : Plots for MSE of proposed work for Speckle and Gaussian Noise

Further, the following figure outlines the contrast study among the present work, algorithm by Liu and present work. The comparison is drawn on the four images for speckle noise. The graph proves that the PSNR and SSIM of the proposed work is higher in all the four cases.
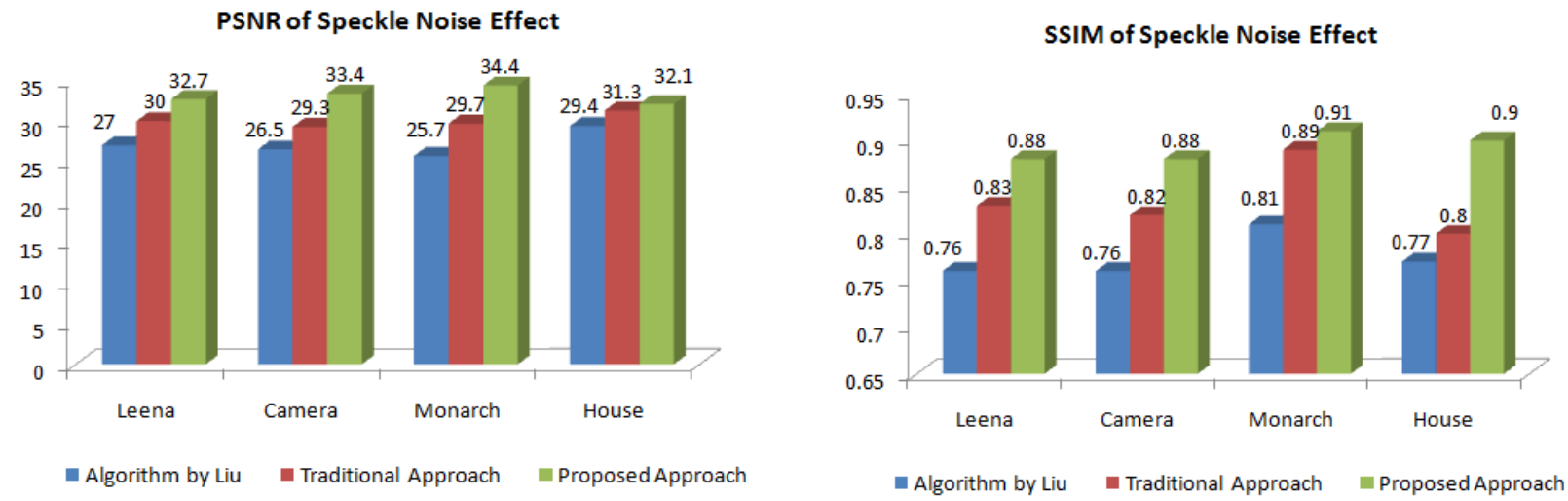

Fig 10 : Plots to compare PSNR and SSIM values of reconstructed general images for three methods 
International Journal of Innovative Technology and Exploring Engineering (IJITEE) ISSN: 2278-3075, Volume-8, Issue-9S, July 2019

\begin{tabular}{|r|l|c|c|c|c|c|c|c|c|c|}
\hline \multirow{2}{*}{ S. NO } & Techniques & Lena & $\begin{array}{l}\text { Cameram } \\
\text { an }\end{array}$ & Monarch & House & Lena & $\begin{array}{l}\text { Cameram } \\
\text { an }\end{array}$ & Monarch & House \\
\cline { 2 - 11 } & \multicolumn{6}{|c|}{ PSNR } \\
\hline 1. & $\begin{array}{l}\text { Algorithm by } \\
\text { Liu }\end{array}$ & 27 & 26.5 & 25.7 & 29.4 & 0.76 & 0.76 & 0.81 & 0.77 \\
\hline 2. & $\begin{array}{l}\text { Traditional } \\
\text { Approach }\end{array}$ & 30 & 29.3 & 29.7 & 31.3 & 0.83 & 0.82 & 0.89 & 0.8 \\
\hline 3. & $\begin{array}{l}\text { Proposed } \\
\text { Approach }\end{array}$ & 32.7 & 33.4 & 34.4 & 32.1 & 0.88 & 0.88 & 0.91 & 0.9 \\
\hline
\end{tabular}

Table 3 : Comparison of PSNR in decibels and SSIM for various general images for three methods

\section{CONCLUSION AND FUTURE SCOPE}

In this era of internet the data travels over then networks in the form of signals whether it is digital or analog. All of the communication channels used for internet based communication leads to the addition of some noisy content in the original data. Thus the introduction of the noise in the original data leads to the alterations in data which modify its original meaning. Thus this study focuses on images that travels over the internet and suffers from the issue of noise. The major intention behind conducting this study along with the outcome can be concluded as follows:

- To analyze the effect of speckle and Gaussian noise on the digital images.

- To apply the Butterworth filters for denoising the images along with coefficients optimization using firefly algorithm.

- To obtain the highest PSNR and SSIM of the resultant images.

In future more enhancements can be done in the present work in following manners:

- Hybrid Filtration can be applied by applying filtration process on both spatial and frequency domain collectively.

\section{REFERENCES}

1. ArchanaMadan, Daljit Singh, “An Optimized Hybrid Filter Designing for Speckle Noise Reduction in Ultrasound Images”, International Journal for Research in Applied Science and Engineering Technology (IJRASET), Vol 5 Issue XII December 2017

2. Rajesh Mohan R, "Speckle Noise Reduction in Images using Wiener Filtering and Adaptive Wavelet Thresholding", IEEE, 2016, Pp 2860-2864

3. Mr. Hitesh, S. Asari, Ami Shah, "A Research Paper On Reducion Of Speckle Noise In Ultrasound Imaging Using Wavelet And Contourlet Transform", Journal of information, knowledge and research in electronics and communication engineering, Vol 2, 2016, Pp 800-806.

4. Milind Kumar V. Sarode, Prashant R. Deshmukh, "Reduction of Speckle Noise and Image Enhancement of Images Using Filtering Technique", International Journal of Advancement in Technology, Vol 2, 2011,

5. AlenrexMaity ;AnshumanPattanaik ; SantwanaSagnika ; SantoshPani, "A Comparative Study on Approaches to Speckle Noise Reduction in Images", IEEE, 2015.

6. T.RathaJeyalakshmi and K.Ramar, "A Modified Method for Speckle Noise Removal in Ultrasound Medical Images", International Journal of Computer and Electrical Engineering, Vol 2, 2010, Pp 1-5.
7. NishthaAttlas, Dr. Sheifali Gupta, "Wavelet Based Techniques for Speckle Noise Reduction in Ultrasound Images", International Journal of Engineering and Research and Application, Vol 4, 2014, Pp 508,513.

8. JyotiJaybhay, RajveerShastri, "speckle noise reduction filters analysis", Research Gate, Vol 2, 2015, Pp 72-78.

9. K.M. SharavanaRaju, Mohammad ShahnawazNasir, T. Meera Devi, "Filtering Techniques to reduce Speckle Noise and Image Quality Enhancement methods on Satellite Images", IOSR-JCE, Vol 15, 2013, Pp 10-15.

10. Karamjeet Singh, SukhjeetKaurRanade, Chandan Singh, "A hybrid algorithm for speckle noise reduction of ultrasound images", ELSEVIER, Vol 148, 2017, Pp 55-69.

11. NagashettappaBiradar, M.L. Dewal, Manoj Kumar Rohit, "Speckle noise reduction in echocardiographic images of aortic valve and cardiac chambers", ELSEVIER, Vol 126, 2015, Pp 153-169.

12. Jian Yang, Jingfan Fan, Yongtian Wang, "Local statistics and non-local mean filter for speckle noise reduction in medical ultrasound image", ELSEVIER, Vol 195, 2016, Pp 88-95.

13. Ajitha R. Subhamathi, "Ultrasound Image Despeckling based on Local Binary Pattern and Local Homogenity", IEEE, 2016, Pp 1758-1761.

14. Jingyun Liu, Zegang Ding, Liangbo Zhao, Fei Dong, Dechengang Liu, "An Adaptive SAR Image Speckle Reduction Algorithm Based on Wavelet Transform and Partial Differential Equations", Proceedings of 10th European Conference on Synthetic Aperture Radar (EUSAR), 2014,Pp 1-4.

\section{AUTHORS PROFILE}

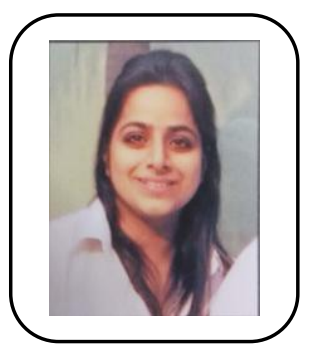

ArchanaMadan

Former Senior Engineer, Max Speciality Films Limited

M.Tech, Guru Nanak Dev Engineering College, Ludhiana, Department of Electronics and Communication Engineering

B.Tech, Lovely Institute of Technology, Jalandhar

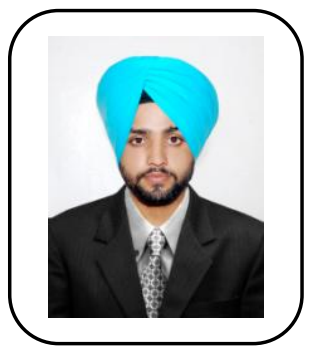

Er. Daljit Singh(Guide)

Assistant Professor - Guru Nanak Dev Engineering College, Department of Electronics and Communication Engineering 